\title{
GDF15 derived from both tumor-associated macrophages and esophageal squamous cell carcinomas contributes to tumor progression via Akt and Erk pathways
}

\author{
Naoki Urakawa ${ }^{1,2}$, Soken Utsunomiya ${ }^{1}$, Mari Nishio ${ }^{1}$, Manabu Shigeoka ${ }^{1,3}$, Nobuhisa Takase ${ }^{1,2}$, Noriaki Arai ${ }^{1}$, \\ Yoshihiro Kakeji ${ }^{2}$, Yu-ichiro Koma' and Hiroshi Yokozaki ${ }^{1}$
}

Tumor-associated macrophages (TAMs) are known to be involved in the progression, angiogenesis, and motility of various cancers. We previously reported the association between an increased number of infiltrating TAMs with tumor progression and poor prognosis in esophageal squamous cell carcinomas (ESCCs). To study the roles of TAMs in ESCC, we first exposed peripheral blood monocyte (PBMo)-derived macrophages from healthy volunteers to conditioned media of TE series human ESCC cell line (TECM) and confirmed the induction of the expression of the M2 macrophage marker CD204 and the protumorigenic factors interleukin (IL)-10, VEGFA, and MMPs. Next, we compared gene expression profiles between PBModerived macrophages stimulated with or without TECM by CDNA microarray and focused on growth differentiation factor 15 (GDF15) among the highly expressed genes including IL-6, IL-8, and CXCL1. Our immunohistochemical study of 70 surgically resected ESCCs revealed that GDF15 was present not only in cancer cells but also in macrophages. The high expression of GDF15 in the ESCCs was significantly correlated with several more malignant phenotypes including vessel invasion, lymph node metastasis, and clinical stages. Patients with high GDF15 expression showed significantly poorer disease-free survival $(P=0.011)$ and overall survival $(P=0.041)$. We also found that recombinant human GDF15 promotes cell proliferation and the phosphorylation of both Akt and Erk1/2 in ESCC cell lines in vitro. These results indicate that GDF15 is secreted by both TAMs and cancer cells in the tumor microenvironment and is associated with aberrant growth and a poor prognosis in human ESCC.

Laboratory Investigation (2015) 95, 491-503; doi:10.1038/labinvest.2015.36; published online 2 March 2015

Tumor microenvironments are composed of neoplastic cells, extracellular matrix, nonmalignant resident stromal cells such as activated fibroblasts, and migratory hemopoietic cells such as macrophages. ${ }^{1}$ Complex interactions among the components of a tumor microenvironment modulate tumor progression, growth, invasion, and angiogenesis. ${ }^{2}$ Macrophages have two different phenotypes, a tumor-suppressive (M1) and a tumor-supportive (M2) phenotype. ${ }^{3}$ M1 macrophages are classically activated by interferon (IFN)- $\gamma$, whereas alternative activation by interleukin (IL)- 4 and IL-13 drives macrophages toward the M2 phenotype. Tumor-associated macrophages (TAMs) are recruited mostly from bone marrow-derived circulating monocytes and they polarize into the M2 or M1/M2 mixed phenotype depending on the microenvironmental factors. ${ }^{4}$ In many cancers, TAMs acquire the M2-like phenotype with IL-10 $0^{\text {high }}$, IL-12 ${ }^{\text {low }}$, cell surface expression of mannose receptors, and scavenger receptors such as CD163 and CD204. ${ }^{4,5}$ In addition, infiltrating TAMs are reported to correlate with poor prognosis by promoting tumor cell growth, invasion angiogenesis, metastasis formation, and immune suppression. ${ }^{6,7}$

Esophageal cancer is the eighth most common cancer worldwide and the sixth leading cause of cancer-related mortality. ${ }^{8}$ An estimated 456000 new esophageal cancer cases (3\% of all cancers) and 0.4 million cancer deaths (5\% of all cancers) were recorded in 2012 worldwide. ${ }^{9}$ The incidence

${ }^{1}$ Division of Pathology, Department of Pathology, Kobe University Graduate School of Medicine, Kobe, Japan; ${ }^{2}$ Division of Gastro-intestinal Surgery, Department of Surgery, Kobe University Graduate School of Medicine, Kobe, Japan and ${ }^{3}$ Division of Oral and Maxillofacial Surgery, Department of Surgery Related, Kobe University Graduate School of Medicine, Kobe, Japan

Correspondence: Professor H Yokozaki, MD, PhD, Division of Pathology, Department of Pathology, Kobe University Graduate School of Medicine, 7-5-1 Kusunoki-cho, Chuoku, Kobe 650-0017, Japan.

E-mail: hyoko@med.kobe-u.ac.jp

Received 3 September 2014; revised 9 December 2014; accepted 9 December 2014 
rate of esophageal cancer, especially of adenocarcinoma, has increased significantly in recent years. ${ }^{10,11}$ As the dominant type of esophageal cancer in Asian countries including Japan, esophageal squamous cell carcinoma (ESCC) has a generally poor prognosis because of the lack of effective clinical methods for its early detection. With regard to epidemiology, smoking and excessive alcohol consumption were reported to increase the risk of ESCC. ${ }^{11}$ Genetic polymorphisms including TP53 and ALDH2 were correlated with an increased risk of ESCC. ${ }^{12,13}$ In the microenvironment of ESCC, Zhang et $a l^{14}$ reported that tumor stroma including cancerassociated fibroblasts were correlated with poor prognosis. However, the pathological mechanisms of TAMs in the tumorigenesis of ESCC remain obscure.

We previously described that the number of infiltrating $\mathrm{CD} 04^{+}$macrophages showed a significant correlation with clinicopathological factors and the prognosis of human ESCC, and that a conditioned medium of the TE series ESCC cell line (TECM) induced CD204 and VEGFA expressions in a differentiated THP-1 human acute monocytic leukemia cell line. ${ }^{15}$ These observations suggested to us that TAMs, mainly expressing CD204, may contribute to the progression of ESCCs whose tumor microenvironment might induce the specific differentiation of macrophages recruited from circulating bone marrow-derived peripheral blood monocytes (PBMos). To test our hypothesis, in the present study we exposed PBMo-derived macrophages from healthy volunteers to TECM and observed the cell's acquisition of TAM-like characteristics. We further investigated the specific cancerassociated gene expression profile in TECM-induced TAM-like macrophages by conducting a cDNA microarray analysis.

\section{MATERIALS AND METHODS Cell Cultures}

Three ESCC cell lines (TE-8, TE-9, and TE-15) were obtained from the RIKEN BioResource Center (Tsukuba, Japan). The individuality of the TE series ESCC cell lines was confirmed by a short tandem repeat analysis at RIKEN and at the Cell Resource Center for Biomedical Research, Institute of Development, Aging and Cancer, Tohoku University (Sendai, Japan). We routinely maintained the ESCC cell lines in RPMI-1640 (Wako, Osaka, Japan) with 10\% fetal bovine serum (FBS) (Sigma-Aldrich, St Louis, MO, USA) and 1\% antibiotic-antimycotic (Invitrogen, Carlsbad, CA, USA). TECMs were prepared by plating $5 \times 10^{6}$ tumor cells in $10 \mathrm{ml}$ complete medium in $100 \mathrm{~mm}$ dishes for $24 \mathrm{~h}$, and thereafter changing the medium to complete DMEM (Wako) supplemented with $10 \%$ human $\mathrm{AB}$ serum (Lonza, Walkersville, MD, USA) instead of FBS. After 2 days, the supernatants were harvested, centrifuged, and stored in aliquots at $-80^{\circ} \mathrm{C}$.

\section{Macrophage Cultures}

Peripheral blood mononuclear cells (PBMCs) were obtained from healthy volunteer donors who provided written informed consent. CD $14^{+}$PBMos were purified from PBMCs by positive selection using the auto MACS Pro Separator (Miltenyi Biotec, Bergisch Gladbach, Germany). PBMos were plated in $1 \times 10^{5}$ cells $/ \mathrm{ml}$ per well, and cultured with macrophage-colony stimulating factor (M-CSF) $(25 \mathrm{ng} / \mathrm{ml}$; R\&D Systems, Minneapolis, MN, USA) for 6 days to induce macrophages differentiation, as described with some modification. ${ }^{16}$ We added $50 \%$ TECM to the cultures for 2 days to induce TAM-like polarization.

\section{Tissue Samples}

A total of 70 sporadic human ESCCs surgically removed from 2005 to 2010 at Kobe University Hospital (Kobe, Japan) were used. The patients were 55 men and 15 women with an age range of 54-88 years and mean age of 65.7 years. None had received adjuvant chemotherapy or radiotherapy before surgery. Informed consent was obtained from all patients, and the study was approved by the Kobe University Institutional Review Board. All resected specimens were fixed in 10\% formalin and embedded in paraffin. Histological and clinicopathological evaluations were performed in accord with the Japanese Classification of Esophageal Cancer proposed by the Japan Esophageal Society along with the TNM classification of the Union for International Cancer Control. ${ }^{17,18}$

\section{Immunohistochemistry}

Immunohistochemistry was performed with labeled streptavidin biotinylated antibody method as previously described. ${ }^{15}$ Specific rabbit polyclonal antibody against growth differentiation factor 15 (GDF15) (HPA011191, 1:50; Sigma-Aldrich, Munich, Germany), specific mouse monoclonal antibodies against CD68 (Kp-1, 1:100; DakoCytomation, Glostrup, Denmark), CD163 (10D6, 1:100; Novocastra, Newcastle upon Tyne, UK), and CD204 (SRA-E5, 1:50; Trans Genic, Kobe, Japan) were used for the primary reaction. For the subsequent reaction, we used labeled streptavidin biotin kit (DakoCytomation). The intensity of GDF15 immunoreactivity in the invasive area of each human ESCC tissue sample was estimated as follows: negative to equal (low) or strong (high), compared with that of the corresponding normal esophageal epithelium. $\mathrm{CD}^{+} 8^{+}, \mathrm{CD} 163^{+}$, and $\mathrm{CD} 204^{+}$cells were counted as macrophages and the estimation was performed as previously described. ${ }^{15}$ Evaluation and scoring were performed by three independent observers (authors NU, YK, and HY).

\section{Immunofluorescence}

Cell and tissue samples were fixed with precooled methanol and then incubated with specific rabbit monoclonal antibody against CD11b (EP1345Y, 1:100; Abcam, Cambridge, UK), rabbit polyclonal antibody against GDF15 (1:50; Sigma-Aldrich), normal rabbit IgG (1:100; Santa Cruz Biotechnology, Dallas, TX, USA), and mouse monoclonal antibodies against CD163 (1:100; Novocastra) and CD204 (1:50; Trans Genic) at $4{ }^{\circ} \mathrm{C}$ overnight. Cy3-conjugated donkey anti-mouse IgG secondary antibody (Jackson ImmunoResearch Laboratories, West Grove, PA, USA), Alexa Fluor-488-conjugated donkey anti-rabbit 
secondary antibody (Jackson ImmunoResearch Laboratories), and DyLight 488-conjugated goat anti-rabbit secondary antibody (Vector Laboratories, Burlingame, CA, USA) were incubated at room temperature for $1 \mathrm{~h}$. The nuclei were stained with DAPI (Wako). Images were taken with a Zeiss LSM 700 laser-scanning microscope and analyzed using the LSM Software ZEN 2009 (Carl Zeiss, Oberkochen, Germany).

\section{Reverse Transcription-PCR (RT-PCR) and Quantitative RT-PCR}

Total mRNA was extracted from each cell using the RNeasy Kit (Qiagen, Hilden, Germany). RT-PCR amplifications of GDF15 and the control gene GAPDH were done. The PCR products were then subjected to electrophoresis in a $2 \%$ agarose gel. Quantitative RT-PCR amplifications of CD163, CD204, IL-10, IL-12p35, VEGFA, MMP2, MMP9, and the control gene GAPDH were performed using the ABI StepOne Real-time PCR system (Applied Biosystems, Foster City, CA, USA). The threshold cycle (Ct) values were determined by plotting the observed fluorescence against the cycle number. $\mathrm{Ct}$ values were analyzed using the comparative threshold cycle method and normalized to those of GAPDH. The relative gene expression levels were estimated using the following formula: relative expression $=2-(\mathrm{Ct}[$ target gene $]-\mathrm{Ct}$ $[G A P D H])$. Primers (CD163, 5'-CGAGTTAACGCCAGT AAG G-3' (forward) and 5'-GAACATGTCACGCCAGC-3' (reverse); CD204, 5'-CCAGGGACATGGGAATGCAA-3' (forward) and 5'-CCAGTGGGACCTCGATCTCC-3' (reverse); IL-10, 5'-GGTTGCCAAGCCTTGTCTGA-3' (forward) and 5'-AGGGAGTTCACATGCGCCT-3' (reverse); IL-12p35, $5^{\prime}$-GCTGGCAGTTATTGATGAGC-3' (forward) and 5'-GCA TGAAGAAGTATGCAGAGC-3' (reverse); VEGFA, 5'-CCTC CGAAACCATGAACTTT-3' (forward) and 5'-TTCTTTGGT CTGCATTCACATT-3' (reverse); MMP2, 5'-ATGGATCCT GGCTTTCCC-3' (forward) and 5'-GCTTCCAAACTTCACG CTG-3' (reverse); MMP9, 5'-ATGCGTGGAGAGTCGAAA TC-3' (forward) and 5'-TACACGCGAGTGAAGGTGAG-3' (reverse); GDF15, 5'-CCCTGCAGTCCGGATACTC-3' (forward) and 5'-GAACAGAGCCCGGTGAAG-3' (reverse); GAPDH, 5'-ACCACAGTCCATGCCATCAC-3' (forward) and $5^{\prime}$-TCCACCACCCTGTTGCTGTA-3' (reverse)) were designed.

\section{cDNA Microarray Analysis}

We extracted total RNA from PBMo-derived macrophages with or without 50\% TECM (TE-8, TE-9, and TE-15) using the RNeasy kit (Qiagen). We prepared a cDNA microarray by using the SurePrint G3 Human GE microarray $8 \times 60 \mathrm{~K}$ v2 (Agilent Technologies, Palo Alto, CA, USA), containing probes for 50599 characterized human genes and expressed sequence tags. We performed in vitro transcription, oligonucleotide array hybridization, and scanning according to the instructions of Takara Bio(Otsu, Japan). Arrays were then scanned with the GeneArray scanner (Agilent Technologies) to obtain image and signal intensities. The data have been deposited to the Gene Expression Omnibus repository (GSE59948).

\section{Western Blotting}

The cells were lysed in a buffer containing $50 \mathrm{mM}$ Tris- $\mathrm{HCl}$ (pH 7.4), $125 \mathrm{mM} \mathrm{NaCl}, 0.1 \%$ Triton $\mathrm{X}-100$, and $5 \mathrm{mM}$ ethylenediaminetetraacetic acid containing both $1 \%$ protease inhibitor and $1 \%$ phosphatase inhibitor cocktail (SigmaAldrich). Proteins were separated by sodium dodecyl sulfatepolyacrylamide gel electrophoresis (SDS-PAGE) followed by electrotransfer onto a polyvinylidene difluoride membrane. After blotting with the following antibodies from Cell Signaling Technology (Beverly, MA, USA) against phosphorylated Akt (Ser473, 1:500), phosphorylated Akt (Thr308, 1:500), non-phosphorylated Akt (1:1000), phosphorylated Erk1/2 (Thr202/Tyr204, 1:200), non-phosphorylated Erk1/2 (1:200), and $\beta$-actin (1:1000), horseradish peroxidase-conjugated anti-mouse or anti-rabbit IgGs (1:1000; GE Healthcare, Little Chalfont, Buckinghamshire, UK) were used as secondary antibodies. The signals were visualized with enhanced chemiluminescence.

\section{Enzyme-Linked Immunosorbent Assay (ELISA)}

PBMo-derived macrophages and ESCC cell lines were cultured in a 24 -well plate $\left(1 \times 10^{5}\right.$ cells per well $)$ with RPMI-1640 plus $10 \%$ FBS and $1 \%$ antibiotic-antimycotic for $48 \mathrm{~h}$. We determined the GDF15 protein levels in the cell culture supernatants by performing the Quantikine ELISA Human GDF15 immunoassay (R\&D Systems) according to the manufacturer's instructions. We determined the optical density of each well using the Microplate Reader Infinite 200 PRO (Tecan, Männedorf, Switzerland) set to $450 \mathrm{~nm}$. Each sample was analyzed in triplicate for statistical analysis. The GDF15 concentration of each sample was calculated using a standard curve and the measured absorbance.

\section{Cell Growth}

After the ESCC cell lines were plated at $5 \times 10^{4}$ cells per $1 \mathrm{ml}$ of serum-free RPMI-1640 overnight, we counted the cells in a Trypan blue dye exclusion assay. Subsequently, the cells were treated with $0,10,50$, and $100 \mathrm{ng} / \mathrm{ml}$ recombinant human GDF15 (rhGDF15; R\&D Systems) for $24 \mathrm{~h}$, and viable cells were also counted. We determined the percentage of cell number in comparison with that of the cells without rhGDF15 treatment.

We also performed an MTS assay (Promega, Madison, WI, USA) to examine the cell growth. $1 \times 10^{5}$ cells in 24 -well plate were incubated with RPMI-1640 serum-free for $24 \mathrm{~h}$. At $24 \mathrm{~h}$ after addition of $100 \mathrm{ng} / \mathrm{ml}$ rhGDF15 (R\&D Systems), we applied CellTiter $96^{\circ}$ AQueous One Solution Reagent $(20 \mu \mathrm{l}$ per $100 \mu \mathrm{l}$ medium; Promega). We determined the optical density of each well using a microplate reader at $490 \mathrm{~nm}$. In a separate set of experiments, we pretreated the ESCC cell lines for $30 \mathrm{~min}$ with a MEK1/2 inhibitor (PD98059, $30 \mu \mathrm{M}$; Cell Signaling Technology) and a PI3K inhibitor (LY294002, 
$20 \mu \mathrm{M}$; Cell Signaling Technology) before adding rhGDF15 for $24 \mathrm{~h}$.

\section{Statistical Analysis}

The results are expressed as mean \pm s.e.m., and statistical significance was analyzed by two-sided Student's $t$-test and one-way ANOVA. The relationships between clinicopathological factors and immunohistochemical results were estimated by $\chi^{2}$ test. Disease-free and overall survival curves were estimated by the Kaplan-Meier method compared by logrank test. Parameters that were significantly associated with disease-free or overall survival rates evaluated in univariate analyses using log-rank tests were further analyzed with multivariate analysis using the Cox proportional hazard regression model. The experimental in vitro data represent two or three independent experiments. A $P$-value of $<0.05$ was considered statistically significant. Statistical analyses were carried out using SPSS Statistics ver. 22 (IBM, Chicago, IL, USA).

\section{RESULTS}

\section{Conditioned Media of ESCC Cell Lines Induced M2-Like} Characteristics in PBMo-Derived Macrophages

We first investigated the expression levels of M2 markers in PBMo-derived macrophages stimulated with 50\% TECM for 2 days in vitro (Figure 1a). Exposure to TECM significantly upregulated the mRNA expression of $I L-10$ and downregulated that of $I L-12 p 35(P<0.05$; Figure $1 b)$. The quantitative RT-PCR and immunofluorescence showed that the expression of both CD163 and CD204 in PBMo-derived macrophages stimulated with TECM were significantly more enhanced than in PBMo-derived macrophages $(P<0.05$; Figures $1 \mathrm{c}$ and $\mathrm{d}$ ). In addition, exposure to TECM significantly enhanced the mRNA expression levels of M2 humoral protumorigenic factors such as VEGFA, MMP2, and MMP9 $(P<0.05$; Figure 1e). Together, these data overall suggest that PBMo-derived macrophages stimulated with TECM acquired M2-like characteristics mimicking those of TAMs.

\section{Upregulation of GDF15 Expression in PBMo-Derived Macrophages Induced by Conditioned Media of ESCC Cell Lines}

We performed a cDNA microarray analysis using total RNAs from PBMo-derived macrophages stimulated with or without TECM to identify any molecules specifically up- or downregulated in PBMo-derived macrophages. After normalization and revision of the raw data, we identified 465 genes $(\log 2$ ratio $>2$ upregulated, 233 genes and $\log 2$ ratio $<-2$ downregulated, 232 genes) as showing significant differences (Supplementary Table 1). The accuracy of the cDNA microarray analysis was confirmed by our quantitative RTPCR analysis of the expression levels of 10 randomly selected differentially expressed genes, as the results showed good concordance with the cDNA microarray data in terms of the $\log 2$ ratio of gene expression. Among the 12 genes related to tumor development (upregulated, seven genes including IL-6, IL-8, and CXCL1; downregulated, five genes; Table 1), we decided to focus on the GDF15 gene transcript (GDB accession no. NM_004864) that showed a $\log 2$ ratio of $\sim 4.0$ in each cell line because TAM-derived GDF15, which is known to be a divergent member of human TGF- $\beta$ superfamily associated with the growth and invasiveness of various cancers, ${ }^{19,20}$ had not yet been reported in the microenvironment of ESCC.

We next investigated whether the induction of the GDF15 expression levels in PBMo-derived macrophages stimulated with TECM was a specific event. We observed a striking upregulation of GDF15 mRNA by TECM in PBMo-derived macrophages along with the induction of M2-like characteristics (Figure 2a). The concentration of secreted GDF15 was significantly higher in the PBMo-derived macrophages stimulated with TE-8CM, TE-9CM, and TE-15CM (1452.0 \pm 49.1 , $482.5 \pm 33.4$, and $414.0 \pm 18.5 \mathrm{pg} / \mathrm{ml}$, respectively) than in the PBMo-derived macrophages $(121.5 \pm 13.8 \mathrm{pg} / \mathrm{ml})$ by ELISA $(P<0.01$; Figure $2 \mathrm{~b})$. Immunofluorescence demonstrated that GDF15 was strongly expressed in the cytoplasm of PBModerived macrophages stimulated with TECM with distinct spindle morphology (Figure 2c). In the ESCC cell lines, the concentration of secreted GDF15 from TE-9 was significantly higher than that of the PBMo-derived macrophages (938.5 \pm 13.9 vs $121.5 \pm 13.8 \mathrm{pg} / \mathrm{ml}, P=0.001)$, whereas GDF15 secretion was undetectable from TE- 8 and TE-15 (Figures $2 a$ and $b$ ).

\section{Not Only Cancer Cells But Also Macrophages Expressed GDF15 in Human ESCC Tissues}

We used immunohistochemistry to examine the expression of GDF15 in human ESCC tissue samples. In the nonneoplastic esophageal tissues, GDF15 was expressed in erythrocytes, normal squamous epithelia, and some stromal cells. In the tumor nests and stroma of human ESCCs, particularly in the invasive area, various levels of GDF15 immunoreactivities were observed: low (i) and high (ii) using the corresponding normal squamous epithelia as positive control (Figure 3a). GDF15 was expressed mostly in cancer cells. In addition, macrophage-like spindle cells with GDF15 immunoreactivity were also found in tumor nests and stroma. Immunofluorescence revealed that a large fraction of $\mathrm{GDF} 15^{+}$spindle cells coexpressed with the M2 macrophage marker CD204 in the tumor nests and stroma of human ESCCs (Figure 3b).

\section{The Expression Levels of GDF15 Were Closely Correlated with Clinicopathological Factors and the Prognosis of the ESCC Patients}

We investigated whether the expression of GDF15 had any statistical association with clinicopathological factors of ESCC patients (Table 2), and we found that a high expression of GDF15 in ESCC was closely correlated with the depth of invasion $(P=0.014)$, lymphatic vessel invasion $(P=0.009)$, blood vessel invasion $(P=0.001)$, lymph node metastasis $(P=0.006)$, and clinical stages $(P=0.001)$. We subsequently 
a

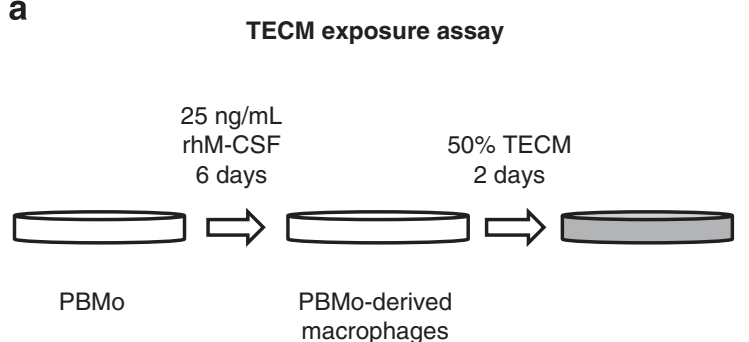

b

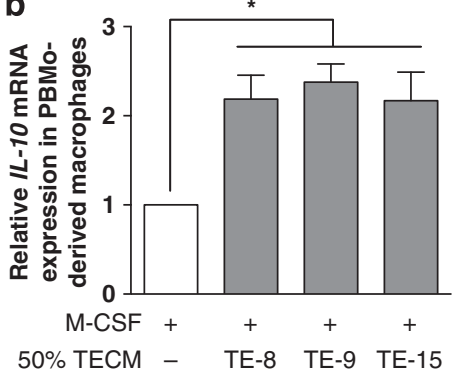

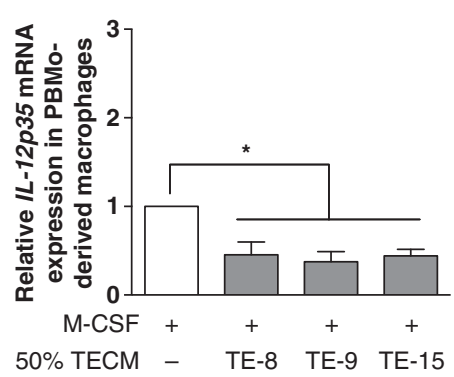

C
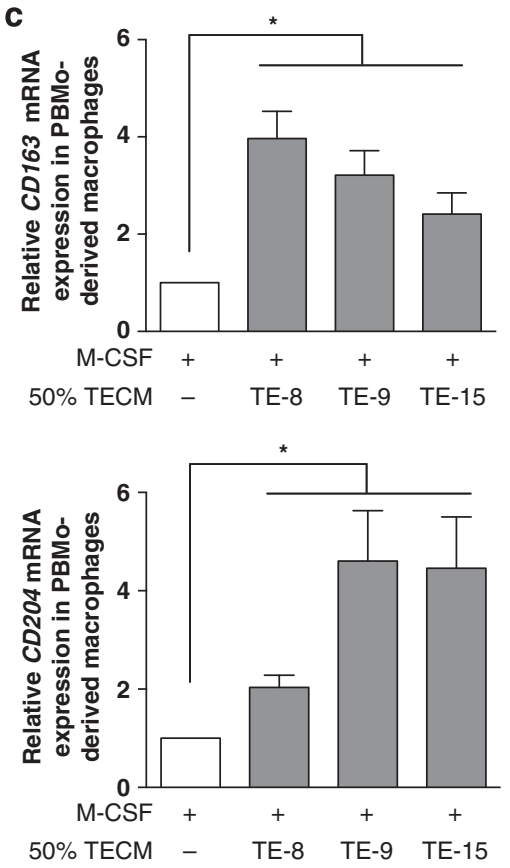

d

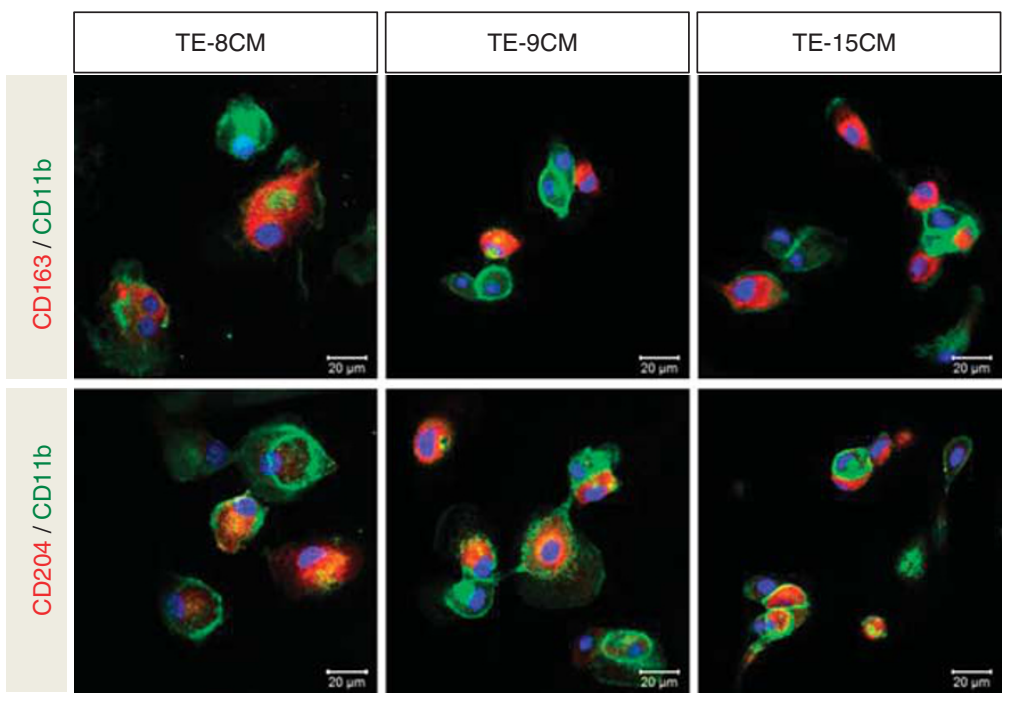

e
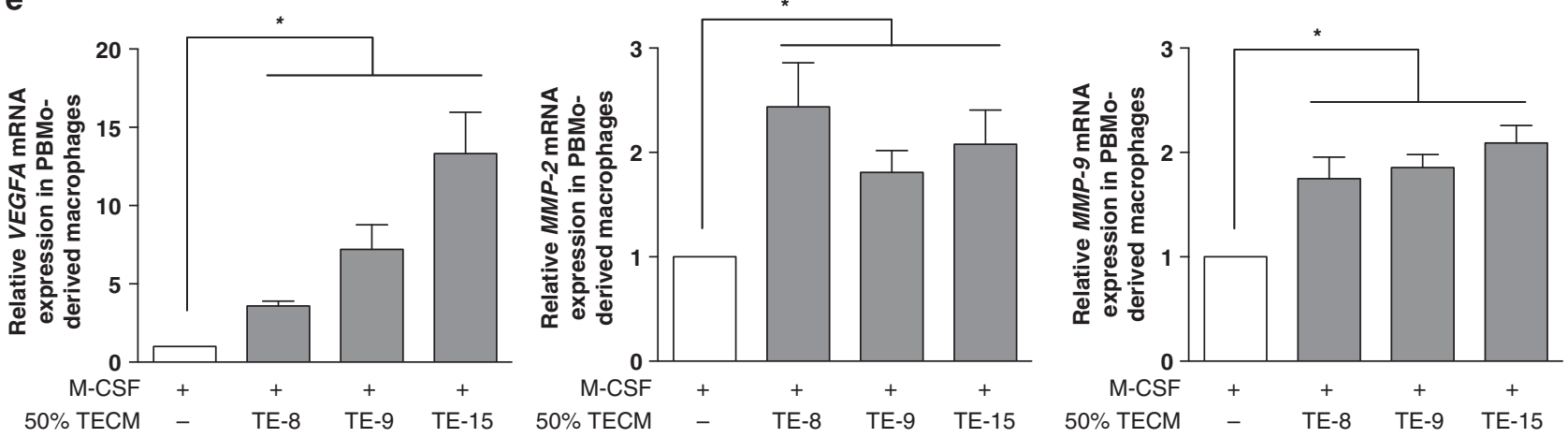

Figure 1 Peripheral blood monocyte (PBMo)-derived macrophages acquired M2-like characteristics by stimulation with conditioned medium of TE series ESCC cell line (TECM). (a) TECM exposure assay: PBMos were treated with $25 \mathrm{ng} / \mathrm{ml}$ recombinant human M-CSF for 6 days to induce macrophage-like differentiation, and then exposed to 50\% TECM for 2 days. (b) IL-10 and IL-12 mRNA expression pattern as M2-like characteristics in PBMo-derived macrophages stimulated with TECM. Expression levels were determined by quantitative RT-PCR and compared with those of PBMo-derived macrophages, normalized to GAPDH expression. Results are mean \pm s.e.m. $\left(n=4 ;{ }^{*} P<0.05\right)$. (c) Induction of M2 markers CD163 and CD204 mRNA in PBMo-derived macrophages stimulated with TECM. Expression levels were analyzed by quantitative RT-PCR. Results are mean \pm s.e.m. ( $n=4 ;{ }^{*} P<0.05$ ). (d) Expression of CD163 and CD204 in PBMo-derived macrophages stimulated with TECM. Double immunofluorescence was performed using anti-CD163 or CD204 (red) plus macrophage marker anti-CD11b (green) in a TECM exposure assay. Coexpression of CD163 and CD204 was detectable in PBModerived macrophages stimulated with TECM. Nuclei were stained with DAPI (blue). Magnification $\times 40$. Scale bar $=20 \mu \mathrm{m}$. (e) Upregulation of protumorigenic factors VEGFA, MMP2, and MMP9. Expression levels were determined by quantitative RT-PCR. Results are mean \pm s.e.m. $\left(n=4 ;{ }^{*} P<0.05\right)$. 
Table 1 Cancer-related genes expressed in PBMo-derived macrophages stimulated with TECM

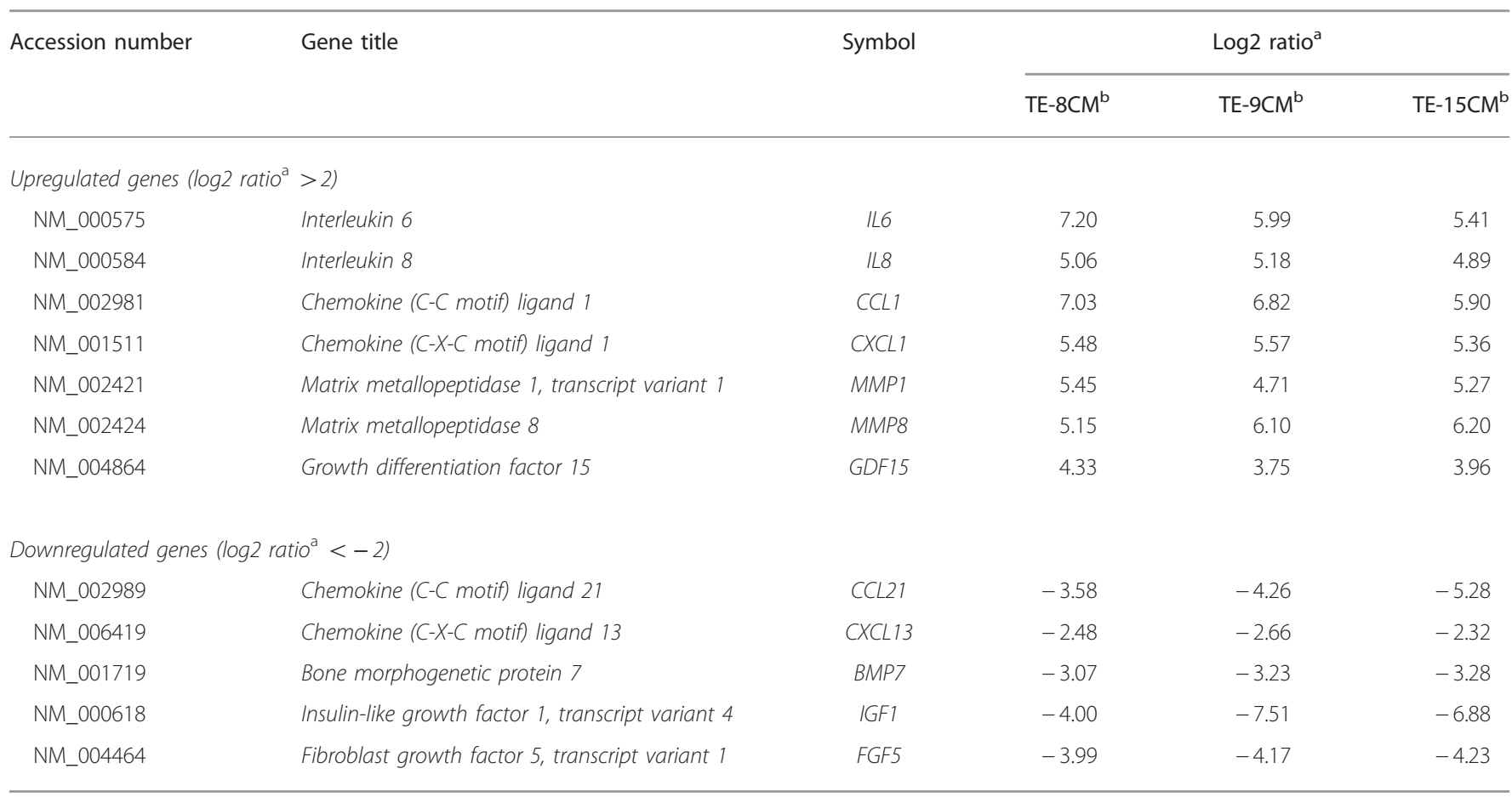

a Log2 ratio: differential expression is reported as the log2 ratio of two spot values in comparison with PBMo-derived macrophages stimulated without TECM. A spot with equal signals on each CDNA microarray have a log2 ratio of zero. ${ }^{\mathrm{b} T E C M}$ : conditioned media of TE series ESCC cell lines.

investigated the association between the expression levels of GDF15 and the number of macrophages in ESCC. We found that a high number of infiltrating $\mathrm{CD}^{+} 8^{+}$or $\mathrm{CD} 163^{+}$ macrophages had a trend to correlate with high expressions of GDF15 $(P=0.025)$. Moreover, high GDF15 expression was significantly associated with a high number of infiltrating CD204 ${ }^{+}$macrophages $(P<0.001$; Table 2$)$.

We next performed a prognostic study of 69 out of 70 ESCC patients (excluding the single patient who could not be followed). The disease-free survival of the patients with a high expression of GDF15 was significantly shorter compared with the patients with low GDF15 by log-rank test (percent disease-free survival after 2 years, 63.0 vs $92.1 \%, P=0.011$; Figure $3 \mathrm{c}$ ). The overall survival of the patients with high GDF15 expression was also significantly shorter than that of the patients with low GDF15 (percent overall survival after 5 years, 59.1 vs $83.1 \%, P=0.041$; Figure 3c). The independent impact of GDF15 on disease-free or overall survival rates was not statistically revealed with multivariate analysis in ESCC (Supplementary Tables 2 and 3).

\section{GDF15 Increased the Growth of the ESCC Cell Lines by Triggering Akt and Erk1/2 Phosphorylation}

We assessed the growth of the ESCC cell lines under serumfree conditions supplemented with titrated concentrations of rhGDF15. We found that GDF15 promoted the proliferation of the ESCC cell lines in a dose-dependent manner after $24 \mathrm{~h}$ : $100 \mathrm{ng} / \mathrm{ml}$ of rhGDF15 yielded up to $141.0 \pm 7.8 \%$ viable TE- 8 cells $(P=0.007), 166.4 \pm 9.3 \%$ viable TE-9 cells $(P<0.001)$, and $141.0 \pm 7.2 \%$ viable TE-15 cells $(P=0.002$; Figure $4 \mathrm{a})$. It has been confirmed that GDF15 activates intracellular signaling cascades such as the PI3K/Akt and MEK/Erk pathways with the induction of the proliferation of cancer cells. ${ }^{19,21}$ We thus wondered whether GDF15 would trigger Akt and Erk1/2 phosphorylation on ESCC cell lines in serumfree conditions. We found that Akt and Erk1/2 phosphorylation in the ESCC cell lines was enhanced with $100 \mathrm{ng} / \mathrm{ml}$ of rhGDF15 after $10 \mathrm{~min}$ (Figure 4b). The GDF15-induced growth for $24 \mathrm{~h}$ in ESCC cells was suppressed by pretreatment for 30 min with a PI3K inhibitor (LY294002, $20 \mu \mathrm{M})$ or a MEK1/2 inhibitor (PD98059, $30 \mu \mathrm{M})(P<0.01$; Figure $4 \mathrm{c}$ ). These results suggested that GDF15 promoted the growth of ESCC cell lines by activating Akt and Erk1/2.

\section{DISCUSSION}

GDF15 is a divergent member of human TGF- $\beta$ superfamily that displays similarity to both classical TGF- $\beta$ isoforms and bone morphogenetic proteins (BMPs). ${ }^{22}$ GDF15, also known as macrophage inhibitory cytokine 1 (MIC-1), nonsteroidal anti-inflammatory drug-activated gene (NAG-1), prostate-derived factor (PDF), placental bone morphogenetic protein (PLAB), and placental TGF- $\beta$ (PTGF- $\beta),{ }^{23-26}$ has 
a

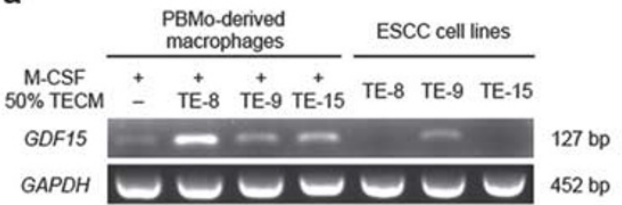

b

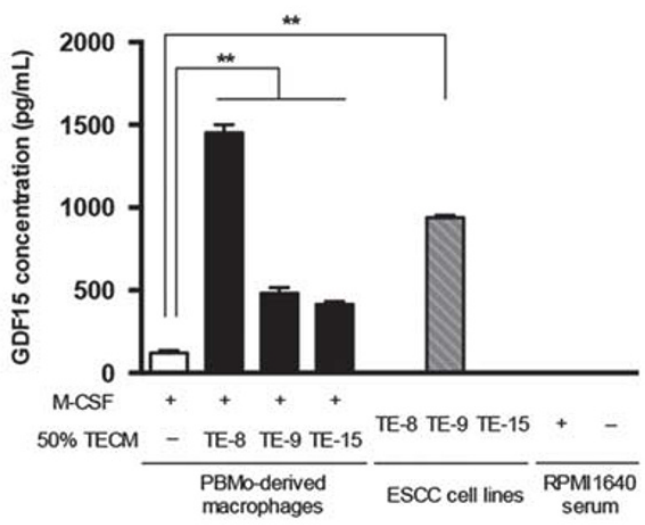

C

(i) PBMo-derived macrophages

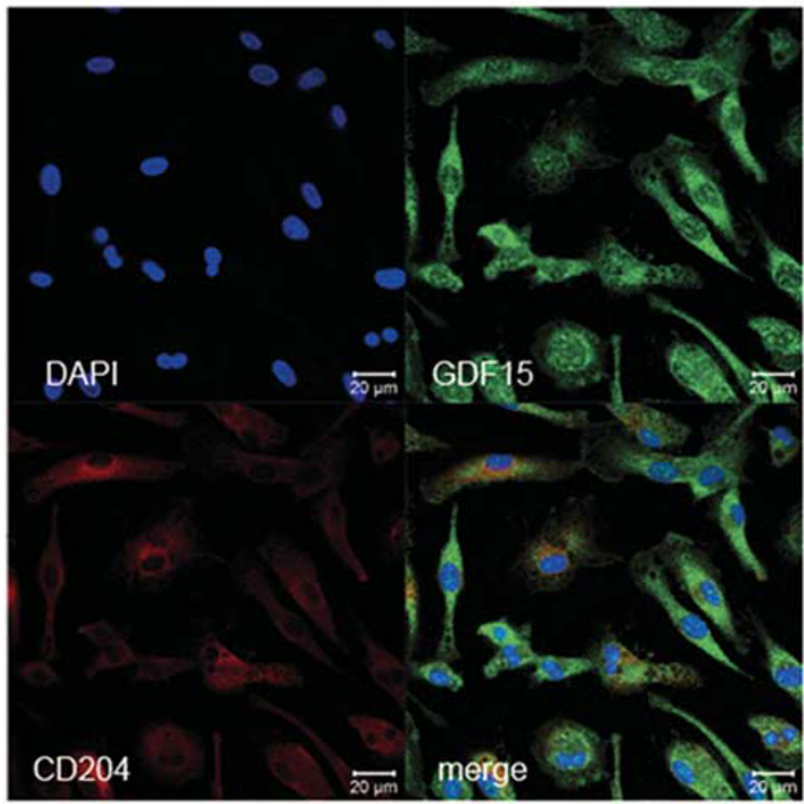

(ii)

PBMo-derived macrophages stimulated with TE-9CM

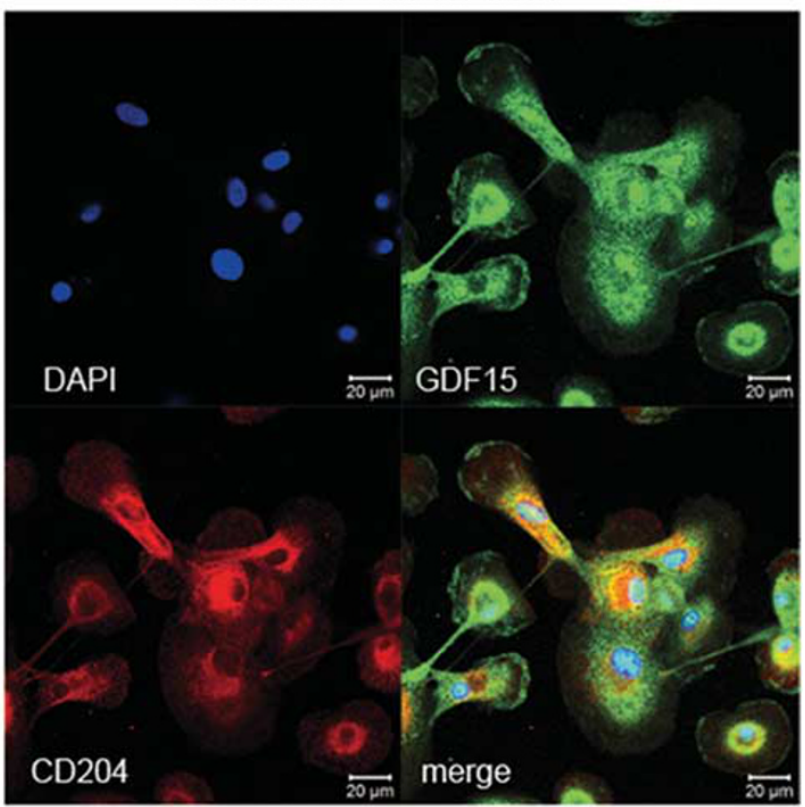

Figure 2 Expression of GDF15 in PBMo-derived macrophages stimulated with TECM and ESCC cell lines. (a) mRNA expression of GDF15 in PBMo-derived macrophages stimulated with TECM and ESCC cell lines. GDF15 and GAPDH primers were used for RT-PCR. The expression levels of GDF15 in the PBModerived macrophages stimulated with TECM were higher than those in the PBMo-derived macrophages. Among the three ESCC cell lines, only TE-9 expressed GDF15. (b) Concentration of GDF15 protein in conditioned medium of PBMo-derived macrophages stimulated with TECM and ESCC cell lines. Protein levels were measured by ELISA. Higher levels of GDF15 secretion were detected in PBMo-derived macrophages stimulated with TECM. The concentration of GDF15 in TE-9 was higher than that in the PBMo-derived macrophages, and that of GDF15 in TE- 8 and TE-15 was undetectable. Data are mean \pm s.e.m. in triplicate $(* * P<0.01)$. (c) Coexpression of GDF15 and CD204 in PBMo-derived macrophages stimulated with TECM. Double immunofluorescence was performed using anti-GDF15 (green) plus anti-CD204 (red) in a TECM exposure assay. The signal intensity of coexpression was more upregulated in PBMo-derived macrophages stimulated with TE-9CM (ii) than in PBMo-derived macrophages (i). Nuclei were stained with DAPI (blue). Magnification $\times 40$. Scale bar $=20 \mu \mathrm{m}$. 
a
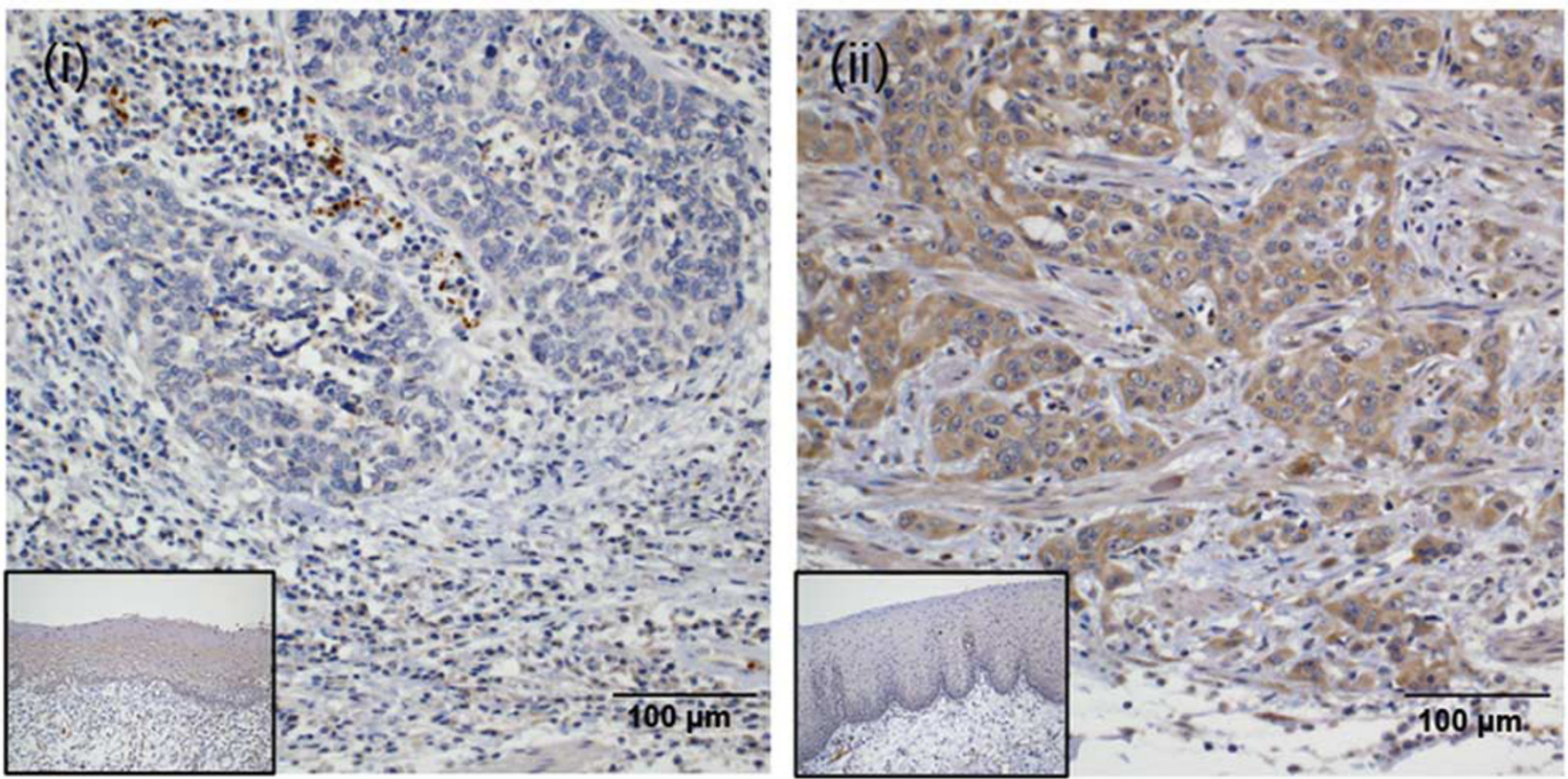

b

(i)
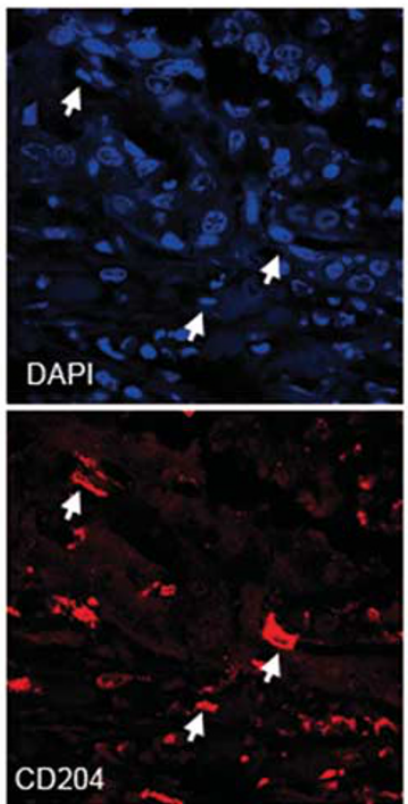
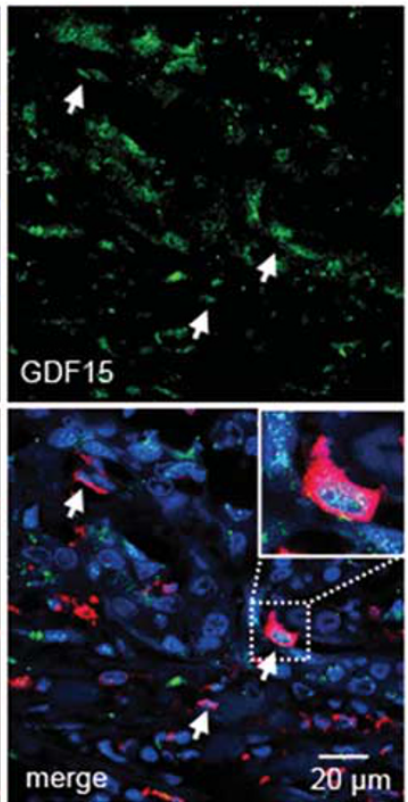

(ii)
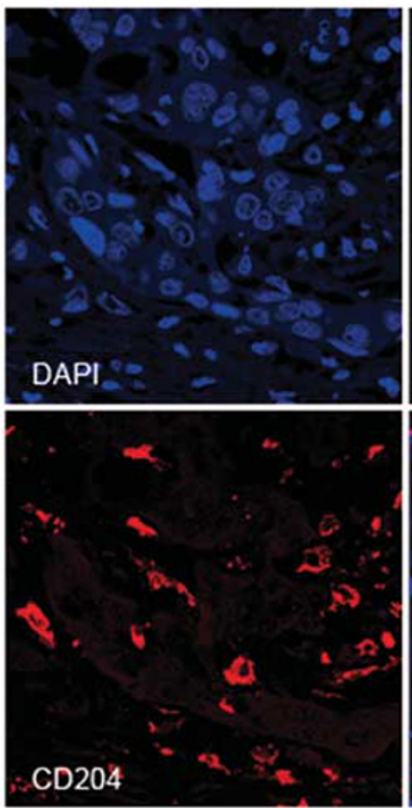
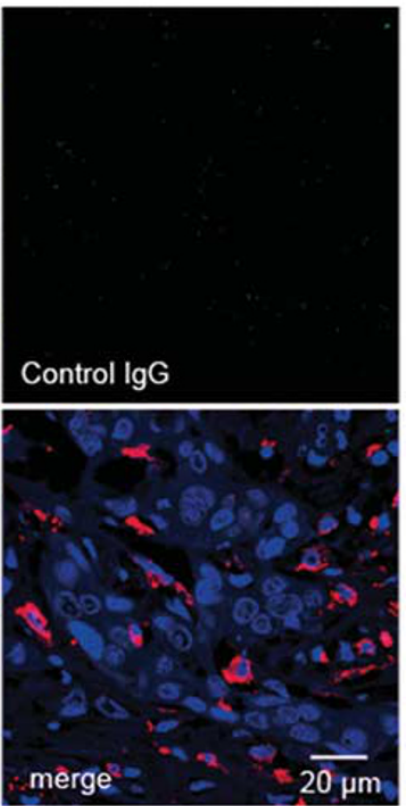

Figure 3 A high expression of GDF15 was detected not only in cancer cells but also in macrophages in the tumor nests and stroma of human ESCC tissues, and it was associated with the prognosis of ESCC patients. (a) Levels of GDF15 in human ESCC tissues by immunostaining: low (i) and high (ii) compared with the corresponding normal esophageal squamous epithelia (insets). Magnification $\times 20$. Scale bar $=100 \mu \mathrm{m}$. (b) GDF15 $/ \mathrm{CD} 204^{+}$ macrophages in the tumor nests and stroma of human ESCC. Double immunofluorescence was performed using anti-GDF15 (green) plus anti-CD204 (red) in a human ESCC tissue sample (i). The arrow indicates GDF15 $/$ CD204 $4^{+}$macrophages in both the tumor nests and stroma of human ESCC tissues. The same area of the tissue sample did not show positive fluorescence by negative control normal lgG (ii). Nuclei were stained with DAPI (blue). Magnification $\times 40$. Scale bar $=20 \mu \mathrm{m}$. Higher magnification of the indicated area (inset). (c) Kaplan-Meier analysis of patients with ESCC. ESCC patients were divided into two groups according to their expression of GDF15; GDF15-low group ( $n=44)$ and GDF15-high group ( $n=25)$. The patients in the GDF15-high group had a significantly poorer disease-free survival (i, 63.0 vs $92.1 \%$ at 2 years, ${ }^{*} P=0.011$ ) and overall survival (ii, 59.1 vs $83.1 \%$ at 5 years, $\left.{ }^{*} P=0.041\right)$. The log-rank test was performed to determine significance. 
c

(i)

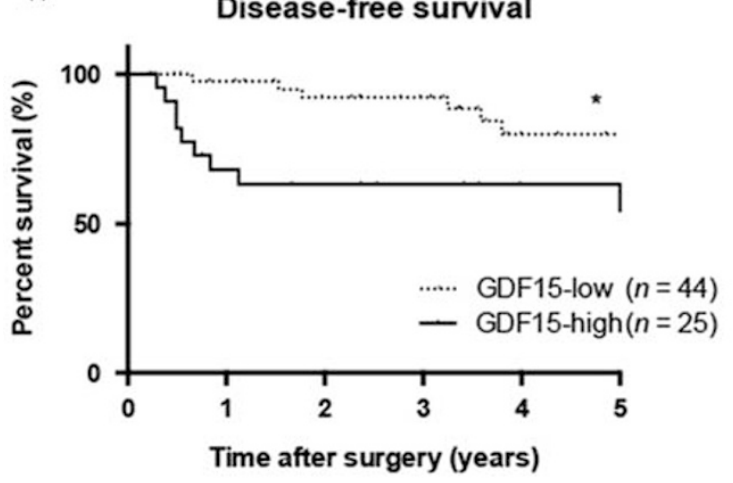

(ii)

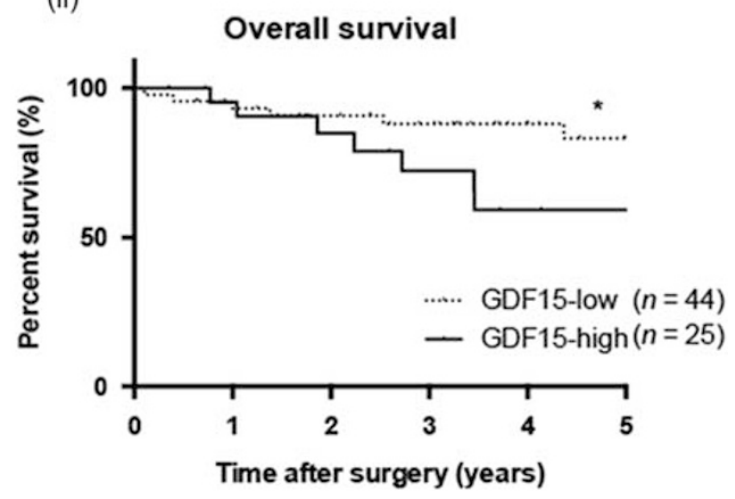

Figure 3 Continued.

broad activity mainly regulating anti-inflammatory and apoptotic pathways. ${ }^{27}$ In healthy subjects, GDF15 is strongly expressed in placenta during pregnancy and at low to moderate levels in brain, liver, breast, colon, and bone marrow. ${ }^{28,29}$ In cancer, GDF15 overexpression has been described in colorectal cancer and malignant glioma. ${ }^{19,30}$ In addition, serum GDF15 levels have been reported to be raised in patients with malignant glioma, pancreatic, or prostate carcinoma compared with those of healthy controls. ${ }^{19,31,32}$ These previous findings may indicate that GDF15 exerts heterogeneous functions in tumors. However, the role of GDF15 in tumorigenesis is still unclear; for instance, a specific receptor for GDF15 has not been identified. ${ }^{33}$ Recent studies suggest that GDF15 might be involved in the growth, survival, and invasiveness of various cancers. ${ }^{19,20,34}$

In the present study, human PBMo-derived macrophages stimulated with TECM acquired M2-like characteristics and upregulated GDF15 in vitro. Several previous studies also showed that PBMo-derived macrophages acquired M2 characteristics such as CD163, CD204, IL-10, VEGFA, and MMPs by conditioned medium of tumor cell line (TCM). ${ }^{5,6,35-37}$ We observed upregulation of various genes in the PBMo-derived macrophages stimulated with TECM by conducting a cDNA microarray analysis. As shown in Table 1 , CXCL1, IL-6, and IL-8 are known to participate in tumor proliferation and angiogenesis. Recent studies have shown that TAM-like PBMo-derived macrophages stimulated with TCM such as those found in breast, ovarian, and colorectal cancer produced these factors. ${ }^{16,38-40}$ In the present study, GDF15 was upregulated in PBMo-derived macrophages stimulated with TECM, whereas, to our knowledge, it has not been reported that TAM-like PBMo-derived macrophages stimulated with other TCMs secreted GDF15. Expression of GDF15 was not detected in cytokine-induced M2-polarized macrophages such as IL-4 and IL-13 (Supplementary Figure 1). Recent studies demonstrated that GDF15 acted as a downstream mediator of the tumor suppressor p53 pathway. ${ }^{33,41}$ As p53 within TAM might contribute to tumor promotion by supporting a paracrine axis of secreted cytokines and chemokines such as IL-6 and CXCL1,38 GDF15 might also be secreted by the same pathway. In addition, we indicated the possibility that GDF15 participated in the macrophage polarization toward a M2 phenotype, especially CD163 induction (Supplementary Figure 2). These data suggest that TAMs have protumorigenic M2-like characteristics in the microenvironment of human ESCC as well as other cancers and may secrete GDF15.

In this study's immunohistochemical investigation, we observed the expression of GDF15 mostly in the invasive area of human ESCC. By conducting an ESCC tissue microarray, Wang et $a l^{42}$ found that normal esophageal epithelium showed negative or weak GDF15 immunoreactions, whereas cancer tissues demonstrated weak to positive staining in 18 of $40(45 \%)$ ESCC specimens. We also found that normal esophageal epithelia showed weak GDF15 immunoreactions, and the expression of GDF15 was mostly stronger in the invasive area of ESCC than at the surface. The invasive area demonstrated strong staining in 25 of our 70 (36\%) ESCC tissue samples compared with the immunoreactions of the corresponding normal esophageal epithelium. These data indicated that GDF15 was present in the microenvironment of human ESCC, especially in the invasive area. In other squamous cell carcinomas, a similar report indicated that normal epithelium showed negative to weak GDF15 immunoreactions and $50 \%$ of oral squamous cell carcinoma tissue also demonstrated strong GDF15 immunoreactivities. ${ }^{43} \mathrm{~A}$ similar upregulation of GDF15 immunoreactivity was also reported in adenocarcinomas including colorectal, gastric, and prostate cancers. ${ }^{30,34,44}$

Wang et $a^{42}$ also indicated that positive staining of GDF15 in ESCC tissues was mostly confined to the cytoplasm of ESCC cells with a diffuse pattern. In the present study too, the GDF15-positive cells in the ESCC tissues were mostly confined to the cytoplasm of ESCC cells, whereas GDF15 immunoreactions were detected in other stromal cells such as erythrocytes and macrophages. Although GDF15 expression 
Table 2 Expression levels of GDF15 in ESCC and their correlation with clinicopathological parameters and infiltrating macrophages phenotypes

Expression of GDF15

\begin{tabular}{|c|c|c|c|c|}
\hline \multicolumn{5}{|l|}{ Age } \\
\hline$<65$ & 33 & 20 & 13 & \multirow[t]{2}{*}{0.544} \\
\hline$\geq 65$ & 37 & 25 & 12 & \\
\hline \multicolumn{5}{|l|}{ Histological grade } \\
\hline HGIEN+WDSCC & 16 & 11 & 5 & \multirow[t]{2}{*}{0.671} \\
\hline MDSCC+PDSCC & 54 & 34 & 20 & \\
\hline \multicolumn{5}{|l|}{ Depth of invasion ${ }^{\mathrm{b}}$} \\
\hline $\mathrm{T} 1$ & 49 & 36 & 13 & \multirow[t]{2}{*}{$0.014^{*}$} \\
\hline $\mathrm{T} 2+\mathrm{T} 3$ & 21 & 9 & 12 & \\
\hline \multicolumn{5}{|c|}{ Lymphatic vessel invasion ${ }^{\mathrm{b}}$} \\
\hline Negative & 37 & 29 & 8 & \multirow[t]{2}{*}{$0.009^{* *}$} \\
\hline Positive & 33 & 16 & 17 & \\
\hline \multicolumn{5}{|c|}{ Blood vessel invasion ${ }^{b}$} \\
\hline Negative & 43 & 34 & 9 & \multirow[t]{2}{*}{$0.001^{* *}$} \\
\hline Positive & 27 & 11 & 16 & \\
\hline \multicolumn{5}{|c|}{ Lymph node metastasis $^{\mathrm{b}}$} \\
\hline Negative & 43 & 33 & 10 & \multirow[t]{2}{*}{$0.006^{* *}$} \\
\hline Positive & 27 & 12 & 15 & \\
\hline \multicolumn{5}{|l|}{ Stage $e^{c}$} \\
\hline $0+1$ & 38 & 31 & 7 & \multirow[t]{2}{*}{$0.001^{* *}$} \\
\hline$\|+I I++I V$ & 32 & 14 & 18 & \\
\hline \multicolumn{5}{|l|}{ CD68 $8^{+}$cells ${ }^{d}$} \\
\hline Low & 35 & 27 & 8 & \multirow[t]{2}{*}{$0.025^{*}$} \\
\hline High & 35 & 18 & 17 & \\
\hline \multicolumn{5}{|l|}{ CD163 ${ }^{+}$cells ${ }^{d}$} \\
\hline Low & 35 & 27 & 8 & \multirow[t]{2}{*}{$0.025^{*}$} \\
\hline High & 35 & 18 & 17 & \\
\hline \multicolumn{5}{|l|}{$\mathrm{CD}_{204^{+}} \mathrm{cells}^{\mathrm{d}}$} \\
\hline Low & 34 & 29 & 5 & \multirow[t]{2}{*}{$<0.001^{* *}$} \\
\hline High & 36 & 16 & 20 & \\
\hline
\end{tabular}

Abbreviations: HGIEN, high-grade intraepithelial neoplasia; MDSCC, moderately differentiated squamous cell carcinoma; PDSCC, poorly differentiated squamous cell carcinoma. T1a, tumor invades mucosa; T1b, tumor invades submucosa; $\mathrm{T} 2$, tumor invades muscularis propria; $\mathrm{T} 3$, tumor invades adventitia; WDSCC, well-differentiated squamous cell carcinoma.

Data were analyzed by $X^{2}$ test and $P<0.05$ was considered statistically significant: ${ }^{*} P<0.05$; ${ }^{* *} P<0.01 ;{ }^{* *} P<0.001$. ${ }^{a}$ Expression of GDF15 in invasive area of human ESCC tissue samples was used to divide the patients into high- and low-expression groups. ${ }^{\text {b}}$ According to the Japanese Classification of Esophageal Cancer. ${ }^{17}$ According to the Tumor Node Metastasis (TNM) classification by Union for International Cancer Control. ${ }^{18}{ }^{\mathrm{d}}$ The median values of $\mathrm{CD}^{2} 8^{+}$, $\mathrm{CD}_{163^{+}}$, or $\mathrm{CD}_{204^{+}}$macrophage numbers in tumor nests and stroma within the areas were used to divide the patients into high and low groups. has been reported in bone marrow stromal cells in multiple myeloma and cancer-associated fibroblasts in prostate cancer, ${ }^{45,46}$ this may be the first report of GDF15 expression in TAMs.

The serum GDF15 concentration has been reported to be closely associated with the depth of invasion, lymph node metastasis, and overall survival of ESCC patients. ${ }^{42,47}$ However, a detailed statistical analysis of the immunohistochemical expression of GDF15 in the ESCC tissues and the clinicopathological factors of the patients had not been described before the present study. We found that the immunoreactivity of GDF15 in the microenvironment of ESCCs was correlated with many clinicopathological factors: the depth of invasion, lymphatic vessel invasion, blood vessel invasion, lymph node metastasis, clinical stages, overall survival, and disease-free survival. In oral and colorectal cancers, several reports have similarly shown that a high expression of GDF15 shown by immunohistochemistry was associated with many tumorigenic factors. ${ }^{30,43}$ These data indicate that GDF15 in the cancer microenvironment might serve as a factor of the tumor progression of human ESCC.

It has been reported that GDF15 induced growth, survival, and invasion of malignant glioma, multiple myeloma, and gastric cancer by activating intracellular signaling cascades such as the PI3K/Akt and MEK/Erk pathways. ${ }^{19,20,34,48}$ Recent studies have also shown that the in vitro proliferation of ESCC cell lines was reduced by inhibition of PI3K or MEK1/2.49,50 Here we demonstrated that GDF15 increased the growth of ESCC cell lines dose-dependently. Moreover, this study is the first to report that both the Akt and Erk1/2 pathways with the induction of proliferation were activated by GDF15 in ESCC cell lines and that treatment with a MEK1/2 inhibitor (PD98059) and a PI3K inhibitor (LY294002) abolished the growth of ESCC cell lines. In addition, indirect co-culture assay revealed the paracrine effects of TAM-derived GDF15 to TE cell lines (Supplementary Figure 3). Our results indicate that GDF15 derived from tumor-associated macrophages and esophageal squamous cell carcinomas might play an important role in growth of ESCC by activating both the PI3K/Akt and MEK/Erk signaling pathways. With regard to GDF15-stimulated invasion, we obtained several positive results indicating GDF15 induced invasion and upregulation of MMPs in TE cell lines by matrigel invasion assay and quantitative RT-PCR in vitro (S Utsunomiya et al, unpublished data). These data also support the clinicopathological result that a high expression of GDF15 by immunohistochemistry is associated with many invasion factors of ESCC.

In summary, the results of the present study indicate that human PBMo-derived macrophages stimulated with TECM acquire M2-like characteristics and upregulate GDF15 expression in human ESCC. Our findings also demonstrate that GDF15 is present in not only cancer cells but also TAMs and that a high expression of GDF15 is closely correlated with a poor prognosis of human ESCC. Both the PI3K/Akt and 

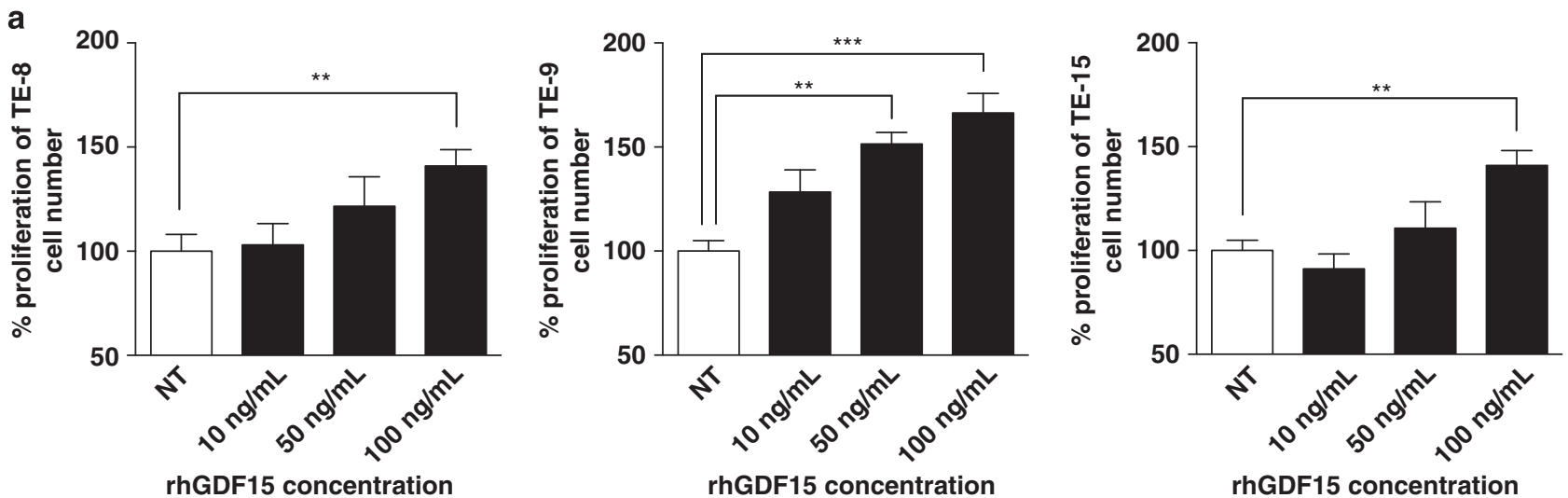

b TE-8

TE-9

Time (min)
rhGDF15 $100 \mathrm{ng} / \mathrm{mL}$
p-Akt (Ser473)
p-Akt (Thr308)
Akt
p-Erk1/2 (Thr202/Tyr204)
Erk1/2
$\beta$-actin
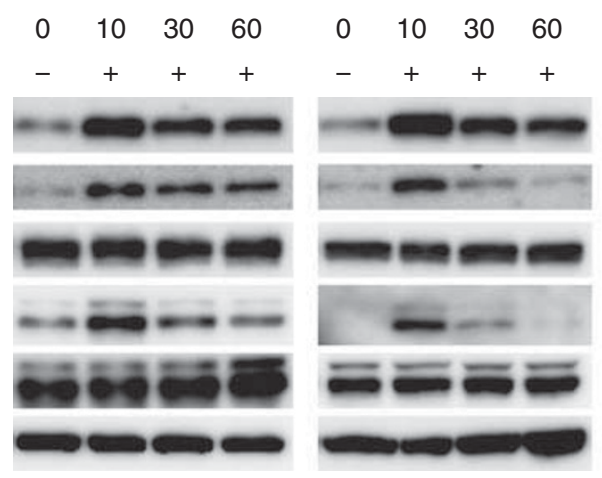

TE-15

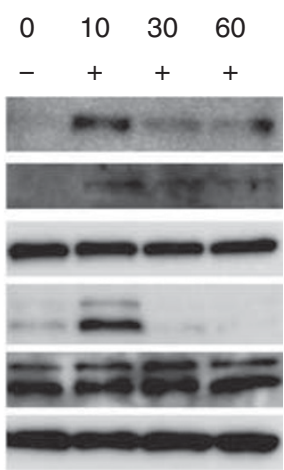

C

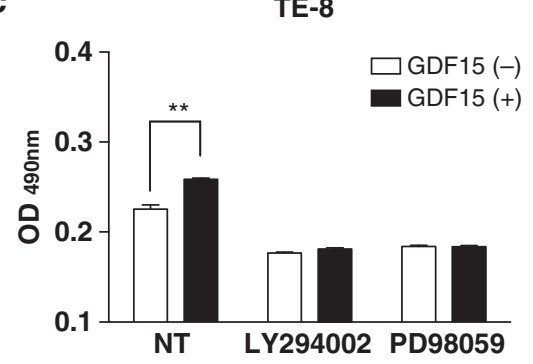

TE-9

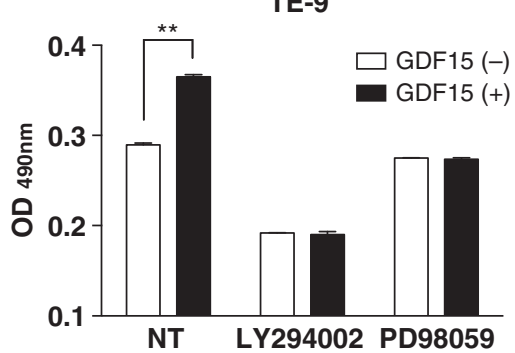

TE-15

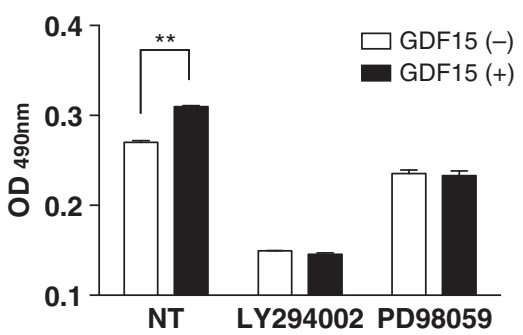

Figure 4 GDF15 promoted the growth of the ESCC cell lines by activating Akt and Erk1/2 phosphorylation. (a) Effect of GDF15 on proliferation in ESCC cell lines. Cells were treated for $24 \mathrm{~h}$ with $\operatorname{rhGDF} 15(10,50$, and $100 \mathrm{ng} / \mathrm{ml})$ under serum-free conditions. The percentage of cell proliferation in controls versus GDF15-treated cells was measured by Trypan blue dye exclusion assay. The growth of ESCC cells was promoted by rhGDF15 dose-dependently. Data are mean \pm s.e.m. from five wells $\left({ }^{*} P<0.01,{ }^{* * *} P<0.001\right)$. NT, no treatment. (b) Phosphorylation of Akt and Erk1/2 in ESCC cell lines by GDF15. TE-8, TE-9, and TE-15 cells were treated with $100 \mathrm{ng} / \mathrm{ml}$ rhGDF15 for $0,10,30$, and 60 min under serum-free conditions. The total proteins were harvested for western blot analysis using specific antibodies against Akt, p-Akt (Ser473), p-Akt (Thr308), Erk1/2, p-Erk1/2 (Thr202/Tyr204), and $\beta$-actin. Not only Erk1/2 but also Akt was activated after $10 \mathrm{~min}$ of rhGDF15 stimulation in ESCC cell lines. (c) The growth-promoting effect of GDF15 on ESCC cell lines was inhibited by LY294002 and PD98059. TE-8, TE-9, and TE-15 were pretreated with a PI3K inhibitor (LY294002), a MEK1/2 inhibitor (PD98059), or none for $30 \mathrm{~min}$ followed by stimulation with $100 \mathrm{ng} / \mathrm{ml}$ rhGDF15 for $24 \mathrm{~h}$ under serum-free conditions. The growth activity of the cells was assessed by the MTS assay. GDF15-induced proliferation was inhibited by both LY294002 and PD98059. Data are mean \pm s.e.m. in triplicate $(* * P<0.01)$.

MEK/Erk pathways with the induction of growth are activated by GDF15 in ESCC cell lines in vitro. GDF15 derived from TAMs and cancer cells in human ESCC may have an important role in the growth and prognosis of human ESCC. It might be suggested that GDF15 is a new biomarker for progression of human ESCC patients.
Supplementary Information accompanies the paper on the Laboratory Investigation website (http://www.laboratoryinvestigation.org)

\section{ACKNOWLEDGMENTS}

This work was supported by Grants-in-Aid for Scientific Research (C)-23590397 and (C)-26460418 from the Japan Society for the Promotion of Science. We thank Dr Shuho Semba, Dr Hiroki Sasaki, and Dr Kazuhiko Aoyagi for valuable 
discussions and technical suggestions about this work. We are also grateful for the excellent technical assistance of Atsuko Kawashima, Yumi Hashimoto, Nobuo Kubo, Miki Yamazaki, and Shuichi Matsumoto.

\section{DISCLOSURE/CONFLICT OF INTEREST}

The authors declare no conflict of interest.

1. Hanahan D, Weinberg RA. Hallmarks of cancer: the next generation. Cell 2011;144:646-674.

2. Balkwill F, Charles KA, Mantovani A. Smoldering and polarized inflammation in the initiation and promotion of malignant disease. Cancer Cell 2005;7:211-217.

3. Mantovani A, Sozzani S, Locati $M$ et al. Macrophage polarization: tumor-associated macrophages as a paradigm for polarized M2 mononuclear phagocytes. Trends Immunol 2002;23:549-555.

4. Mantovani A, Schioppa T, Porta C et al. Role of tumor-associated macrophages in tumor progression and invasion. Cancer Metastasis Rev 2006:25:315-322.

5. Hagemann T, Wilson J, Burke $F$ et al. Ovarian cancer cells polarize macrophages toward a tumor-associated phenotype. J Immunol 2006;176:5023-5032.

6. Komohara Y, Ohnishi K, Kuratsu J et al. Possible involvement of the M2 anti-inflammatory macrophage phenotype in growth of human gliomas. J Pathol 2008;216:15-24.

7. Hasita $\mathrm{H}$, Komohara $\mathrm{Y}$, Okabe $\mathrm{H}$ et al. Significance of alternatively activated macrophages in patients with intrahepatic cholangiocarcinoma. Cancer Sci 2010;101:1913-1919.

8. Zhu HD, Guo JH, Mao AW et al. Conventional stents versus stents loaded with 125iodine seeds for the treatment of unresectable oesophageal cancer: a multicentre, randomised phase 3 trial. Lancet Oncol 2014;15:612-619.

9. Montgomery EA, Basman FT, Brennan $\mathrm{P}$ et al. Oesophageal caner. In: Stewart BW, Wild CP (eds). World Cancer Report 2014. IARC: Lyon, 2014, pp 374-382.

10. Jemal A, Murray T, Ward E et al. Cancer statistics, 2005. CA Cancer J Clin 2005:55:10-30.

11. Jemal A, Bray F, Center MM et al. Global cancer statistics. CA Cancer J Clin 2011;61:69-90.

12. Hiyama T, Yoshihara M, Tanaka $S$ et al. Genetic polymorphisms and esophageal cancer risk. Int J Cancer 2007;121:1643-1658.

13. Umar SB, Fleischer DE. Esophageal cancer: epidemiology, pathogenesis and prevention. Nat Clin Pract Gastroenterol Hepatol 2008;5: 517-526.

14. Zhang C, Fu L, Fu J et al. Fibroblast growth factor receptor 2-positive fibroblasts provide a suitable microenvironment for tumor development and progression in esophageal carcinoma. Clin Cancer Res 2009;15:4017-4027.

15. Shigeoka M, Urakawa N, Nakamura T et al. Tumor associated macrophage expressing CD204 is associated with tumor aggressiveness of esophageal squamous cell carcinoma. Cancer Sci 2013;104: 1112-1119.

16. Solinas G, Schiarea S, Liguori M et al. Tumor-conditioned macrophages secrete migration-stimulating factor: a new marker for M2-polarization influencing tumor cell motility. J Immunol 2010;185:642-652.

17. Japan Esophageal Society. Japanese Classification of Esophageal Cancer10th ednKanehara \& Co: Tokyo, 2008.

18. Sobin LH, Gospodarowicz MK, Wittekind C (eds). TNM Classification of Malignant Tumours7th ednWiley-Blackwell: Hoboken, NJ, 2011; 336p.

19. Roth $\mathrm{P}$, Junker $\mathrm{M}$, Tritschler I et al. GDF-15 contributes to proliferation and immune escape of malignant gliomas. Clin Cancer Res 2010;16: 3851-3859.

20. Corre J, Labat E, Espagnolle $\mathrm{N}$ et al. Bioactivity and prognostic significance of growth differentiation factor GDF15 secreted by bone marrow mesenchymal stem cells in multiple myeloma. Cancer Res 2012;72:1395-1406.

21. Griner SE, Joshi JP, Nahta R. Growth differentiation factor 15 stimulates rapamycin-sensitive ovarian cancer cell growth and invasion. Biochem Pharmacol 2013;85:46-58.
22. Bootcov MR, Bauskin AR, Valenzuela SM et al. MIC-1, a novel macrophage inhibitory cytokine, is a divergent member of the TGFbeta superfamily. Proc Natl Acad Sci USA 1997:94:11514-11519.

23. Baek SJ, Horowitz JM, Eling TE. Molecular cloning and characterization of human nonsteroidal anti-inflammatory drug-activated gene promoter. Basal transcription is mediated by $\mathrm{Sp} 1$ and Sp3. J Biol Chem 2001;276:33384-33392.

24. Paralkar VM, Vail AL, Grasser WA et al. Cloning and characterization of a novel member of the transforming growth factor-beta/ bone morphogenetic protein family. J Biol Chem 1998;273: 13760-13767.

25. Hromas $R$, Hufford $M$, Sutton J et al. PLAB, a novel placental bone morphogenetic protein. Biochim Biophys Acta 1997;1354:40-44.

26. Li PX, Wong J, Ayed A et al. Placental transforming growth factor-beta is a downstream mediator of the growth arrest and apoptotic response of tumor cells to DNA damage and p53 overexpression. J Biol Chem 2000;275:20127-20135.

27. Ago T, Sadoshima J. GDF15, a cardioprotective TGF-beta superfamily protein. Circ Res 2006;98:294-297.

28. Yokoyama-Kobayashi $M$, Saeki $M$, Sekine $S$ et al. Human CDNA encoding a novel TGF-beta superfamily protein highly expressed in placenta. J Biochem 1997;122:622-626.

29. Strelau J, Sullivan A, Bottner $M$ et al. Growth/differentiation factor-15/ macrophage inhibitory cytokine- 1 is a novel trophic factor for midbrain dopaminergic neurons in vivo. J Neurosci 2000;20: 8597-8603.

30. Wallin $\mathrm{U}$, Glimelius B, Jirstrom $\mathrm{K}$ et al. Growth differentiation factor 15 : a prognostic marker for recurrence in colorectal cancer. Br J Cancer 2011;104:1619-1627.

31. Koopmann J, Rosenzweig CN, Zhang Z et al. Serum markers in patients with resectable pancreatic adenocarcinoma: macrophage inhibitory cytokine 1 versus CA19-9. Clin Cancer Res 2006;12:442-446.

32. Brown DA, Lindmark F, Stattin $P$ et al. Macrophage inhibitory cytokine 1: a new prognostic marker in prostate cancer. Clin Cancer Res 2009:15:6658-6664

33. Wang $X$, Baek SJ, Eling TE. The diverse roles of nonsteroidal antiinflammatory drug activated gene (NAG-1/GDF15) in cancer. Biochem Pharmacol 2013;85:597-606.

34. Lee $D H$, Yang $Y$, Lee $S J$ et al. Macrophage inhibitory cytokine-1 induces the invasiveness of gastric cancer cells by up-regulating the urokinase-type plasminogen activator system. Cancer Res 2003;63: 4648-4655.

35. Komohara $\mathrm{Y}$, Hasita $\mathrm{H}$, Ohnishi $\mathrm{K}$ et al. Macrophage infiltration and its prognostic relevance in clear cell renal cell carcinoma. Cancer Sci 2011;102:1424-1431.

36. $\mathrm{Xu} \mathrm{W}$, Schlagwein $\mathrm{N}$, Roos $\mathrm{A}$ et al. Human peritoneal macrophages show functional characteristics of M-CSF-driven anti-inflammatory type 2 macrophages. Eur J Immunol 2007;37:1594-1599.

37. Helm O, Held-Feindt J, Grage-Griebenow E et al. Tumor-associated macrophages exhibit pro- and anti-inflammatory properties by which they impact on pancreatic tumorigenesis. Int J Cancer 2014;135: 843-861.

38. Lowe JM, Menendez D, Bushel PR et al. p53 and NF-kappaB coregulate proinflammatory gene responses in human macrophages. Cancer Res 2014;74:2182-2192.

39. Allavena P, Mantovani A. Immunology in the clinic review series; focus on cancer: tumour-associated macrophages: undisputed stars of the inflammatory tumour microenvironment. Clin Exp Immunol 2012;167: 195-205.

40. Grugan KD, McCabe FL, Kinder M et al. Tumor-associated macrophages promote invasion while retaining Fc-dependent anti-tumor function. J Immunol 2012;189:5457-5466.

41. Mimeault M, Batra SK. Divergent molecular mechanisms underlying the pleiotropic functions of macrophage inhibitory cytokine-1 in cancer. J Cell Physiol 2010;224:626-635.

42. Wang $X B$, Jiang $X R, Y u X Y$ et al. Macrophage inhibitory factor 1 acts as a potential biomarker in patients with esophageal squamous cell carcinoma and is a target for antibody-based therapy. Cancer Sci 2014;105:176-185.

43. Zhang L, Yang X, Pan HY et al. Expression of growth differentiation factor 15 is positively correlated with histopathological malignant grade and in vitro cell proliferation in oral squamous cell carcinoma. Oral Oncol 2009;45:627-632. 
44. Karan D, Chen SJ, Johansson SL et al. Dysregulated expression of MIC-1/PDF in human prostate tumor cells. Biochem Biophys Res Commun 2003;305:598-604.

45. Tanno T, Lim Y, Wang Q et al. Growth differentiating factor 15 enhances the tumor-initiating and self-renewal potential of multiple myeloma cells. Blood 2014;123:725-733.

46. Bruzzese $F$, Hagglof $C$, Leone $A$ et al. Local and systemic pro-tumorigenic effects of fibroblast-derived GDF15/MIC-1. Cancer Res 2014;74: 3408-3417.

47. Skipworth RJ, Deans DA, Tan BH et al. Plasma MIC-1 correlates with systemic inflammation but is not an independent determinant of nutritional status or survival in oesophago-gastric cancer. Br J Cancer 2010;102:665-672.

48. Si $Y$, Liu $X$, Cheng $M$ et al. Growth differentiation factor 15 is induced by hepatitis $C$ virus infection and regulates hepatocellular carcinomarelated genes. PLoS One 2011;6:e19967.

49. Li B, Tsao SW, Li YY et al. Id-1 promotes tumorigenicity and metastasis of human esophageal cancer cells through activation of PI3K/AKT signaling pathway. Int J Cancer 2009;125:2576-2585.

50. Liu F, Zheng S, Liu T et al. MicroRNA-21 promotes the proliferation and inhibits apoptosis in Eca109 via activating ERK1/2/MAPK pathway. Mol Cell Biochem 2013;381:115-125. 